\title{
Different solid sample preparation methods affecting the spectral similarity of salmon calcitonin
}

\author{
Shan-Yang Lin *, Chih-Cheng Lin and Ting-Huei Lee \\ Department of Biotechnology, Yuanpei University, Hsin Chu, Taiwan
}

\begin{abstract}
Salmon calcitonin (sCT) was selected as a model protein drug for investigating its structural similarity in the solid state by four sample preparation methods, such as tape, smeared, $\mathrm{CaF}_{2}$ and film methods. The conformational changes of sCT in the solid state were estimated by using a second-derivative Fourier transform infrared (FT-IR) microspectroscopy. The tape method was acted as a standard reference.

The value of correlation coefficient $(r)$ for smeared method was higher than that of other method, indicating that a novel technique by smearing SCT powder on the surface of $\mathrm{KBr}$ pellet was the best optimal sample preparation method.
\end{abstract}

Keywords: Salmon calcitonin, FT-IR, sample preparation, second derivative, structural similarity

\section{Introduction}

The therapeutic activity of protein drug is well known to be highly dependent on their conformational structure, how to keep the structural integrity and active conformation of protein drug in the manufacturing processes of production, or shipping and long-term storage of products are the highlighted and critical issues [4,7]. Because the secondary structure is one of the most important conformational information for a protein, thus the secondary structure prediction and determination of proteins are the important events $[10,20,25]$. Many analytical techniques have been applied to determine the secondary structure of protein $[18,22,23]$, Fourier transform infrared (FT-IR) spectroscopy is one of the most common spectroscopic techniques to quickly examine the conformational changes of protein structure in the solid and liquid states $[1,8,16]$.

Since protein drug formulations are more stable in the solid state than in the liquid state, the solid-state protein products have often been manufactured [8,15,24]. Before FT-IR determination of protein secondary conformation in the solid state, the solid-state protein sample was commonly mixed and ground with $\mathrm{KBr}$ powder, and then compressed in a mechanical die press to form a translucent pellet. These two processes of grinding and compression might cause the additional protein structural alterations [6,14], implying that the sample preparation method for protein in the solid state plays an important role during FT-IR determination. In order to avoid both processing effects, a unique solid sample preparation method is needed.

\footnotetext{
${ }^{*}$ Corresponding author: Prof. Shan-Yang Lin, PhD, Lab. Pharm. Biopharm., Department of Biotechnology, Yuanpei University, Hsin Chu, Taiwan. Tel.: +886 03 5381183 8157; Fax: +886 03 6102328; E-mail: sylin@ mail.ypu.edu.tw.
} 
Calcitonin (CT), a 32-amino acid linear polypeptide hormone, is always used for the therapy of different bone diseases [2,17]. Among different types of CT available for clinic use, salmon calcitonin (sCT) is one of the most potent forms [2,9]. Very little has been reported regarding the structural stability of sCT formulations in the solid state. In this study, we used sCT as a model protein drug to preliminarily examine the influence of different sample preparation methods on the conformational structure of $\mathrm{sCT}$ in the solid state.

\section{Materials and methods}

\subsection{Materials}

Salmon calcitonin (sCT) was purchased from Polypeptide laboratory A/S, Denmark. The KBr crystals were obtained from Jasco Parts Center (Jasco Co., Tokyo, Japan). Aluminum foil was purchased from Reynolds Metals (VA, USA).

\subsection{Sample preparation methods}

(1) Tape method: A tiny sample of sCT powder was partly adhered with adhesive tape and fixed on the edge of glass plate.

(2) Smeared method: A trace amount of sCT powder was carefully smeared on the surface of $\mathrm{KBr}$ pellet without any compression.

(3) $\mathrm{CaF}_{2}$ method: One drop of $1 \%$ (w/v) sCT aqueous solution was dropped on the surface of $\mathrm{CaF}_{2}$ plate and stored at $25^{\circ} \mathrm{C}, 50 \%$ relative humidity $(\mathrm{RH})$ condition. After storage for 1 day, the cast film on the foil was formed.

(4) Film method: One drop of $1 \%(\mathrm{w} / \mathrm{v}) \mathrm{sCT}$ aqueous solution was dropped on the aluminum foil and stored at $25^{\circ} \mathrm{C}, 50 \%$ relative humidity $(\mathrm{RH})$ condition. The cast film on the foil was formed after storage for 1 day.

All the solid samples were stored at $25 \pm 2{ }^{\circ} \mathrm{C}, 60 \pm 5 \% \mathrm{RH}$ condition before spectral analysis.

\subsection{FT-IR microspectroscopic studies of different sCT samples}

Each IR spectrum of sCT sample prepared by tape, smeared or $\mathrm{CaF}_{2}$ method was determined by FTIR microspectroscopy (Micro FTIR-200, Jasco Co., Japan) equipped with a mercury cadmium telluride (MCT) detector using a transmission technique, according to our previous studies [11,12]. All the spectra were obtained at a $4 \mathrm{~cm}^{-1}$ resolution and at 100 scans. However, the IR spectrum of sCT film prepared by a film method was also determined by using a reflectance technique [13]. The reflectance IR spectra were collected at an angle of incidence centered at $30^{\circ}$. All the determinations were undertaken at $25 \pm 2{ }^{\circ} \mathrm{C}$ and $60 \pm 5 \%$ RH condition.

\subsection{Data acquisition and handling}

\subsubsection{Spectral analysis}

A software of spectral manager for window (Jasco Co., Tokyo, Japan) and GRAMS spectroscopy software suite (Version 7, Thermo Electron Co., MA, USA) were used for data acquisition and handling. Second-derivative spectral analysis was applied to locate the position of the overlapping components in the amide bands and assigned to different secondary structures [21]. 


\subsubsection{Structural similarity}

In order to quantify the structural similarity of sCT samples prepared by different methods, the spectral correlation coefficient analysis between two second-derivative IR spectra was applied. A mathematical procedure proposed by Prestrelski et al. was used to calculate the spectral correlation coefficient $(r)$ between two second-derivative IR spectra as follow [19]:

$$
r=\frac{\sum^{n} x_{i} y_{i}}{\sqrt{\sum x_{i}^{2} y_{i}^{2}}}
$$

where $x_{i}$ and $y_{i}$ are the spectral absorbance values of the reference and comparison spectra respectively, at the $i$ th frequency position in the amide I region.

The tape method was acted as a standard reference, since this method maintained a native form of sCT without any treatment. The $r$ value provides a measure to compare each IR spectrum of a given sCT sample prepared by one of different methods to that of sCT sample prepared by a tape method. All the spectral comparisons were performed in the amide I region $\left(1700-1600 \mathrm{~cm}^{-1}\right)$. Each spectrum was baseline-offset corrected and area-normalized. Comparison of identical spectra gives a value of 1.0. The larger the changes in conformation detected, the greater the difference between two spectra obtained, leading to a smaller $r$ value.

\section{Results and discussion}

Infrared spectra of sCT sample prepared by four different methods (tape, smeared, $\mathrm{CaF}_{2}$ and film) within the range of 3700-2800 and 1800-1000 $\mathrm{cm}^{-1}$, are shown in Fig. 1. It is evident that all the IR spectra from four sample preparation methods seemed to be similar. The peaks at $3296-3230 \mathrm{~cm}^{-1}$ were originated from the vibrations of NH stretching mode of sCT. The peaks near $2957-2959 \mathrm{~cm}^{-1}$ were due to the asymmetric $\mathrm{CH}_{3}$ and $\mathrm{CH}_{2}$ stretching bands of sCT, while the peaks at $2871 \mathrm{~cm}^{-1}$ was associated

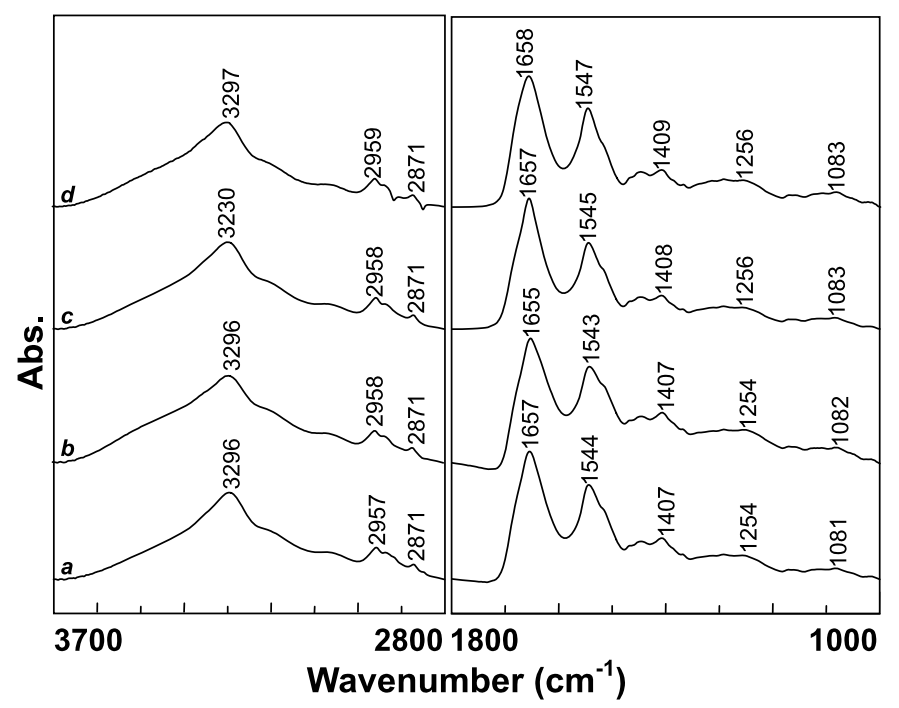

Fig. 1. Infrared spectra of sCT prepared by four different methods within the range of 3700-2800 and 1800-1000 $\mathrm{cm}^{-1}$. Key: (a) tape method; (b) smeared method; (c) $\mathrm{CaF}_{2}$ method; (d) film method. 
with the symmetric $\mathrm{CH}_{3}$ stretching mode of the side chains of sCT. The amide I, II and III bands were located at $1657-1658,1544-1547$ and $1254-1256 \mathrm{~cm}^{-1}$, respectively. The peaks at $1407-1409 \mathrm{~cm}^{-1}$ were due to the symmetric $\mathrm{COO}^{-}$stretching band and/or the deformation of $\mathrm{CH}_{2}$ and $\mathrm{CH}_{3}$. The shoulders at $1081-1083 \mathrm{~cm}^{-1}$ were mainly corresponded to the contributions of $\mathrm{C}-\mathrm{O}$ and $\mathrm{C}-\mathrm{N}$ stretching modes [3,5]. Because all samples exhibited the similar IR spectra, suggesting that four sample preparation methods did not markedly alter the conformational structure of sCT in the solid state.

The amide I band in the IR spectrum is particularly more sensitive to protein secondary structure than other amide bands, since the amide $\mathrm{I}$ band arising from the $\mathrm{C}=\mathrm{O}$ and $\mathrm{N}-\mathrm{H}$ groups is susceptible to hydrogen bonding and coupling between transition dipole of adjacent peptide bonds in the structure of proteins. Thus the amide I band is the most suitable probe used to differentiate different secondary structures of protein. The second-derivative spectra have been applied to resolve the overlapping bands within the amide I band and to express their secondary structure [21]. Since the height of a second-derivative IR peak is proportional to the square of the original peak height with an opposite sign, the position and height of a second-derivative peak may quantitatively reflect the secondary structure of SCT. To further verify the structural similarity of sCT prepared by different sample preparation methods, a spectral correlation coefficient analysis between two second-derivative amide I spectra was carried out. The IR spectrum of sCT prepared by tape method was compared with that of the IR spectrum of sCT prepared by smeared, $\mathrm{CaF}_{2}$ or film method using the second-derivative spectra in amide I region (Fig. $2 \mathrm{~A}$ ).

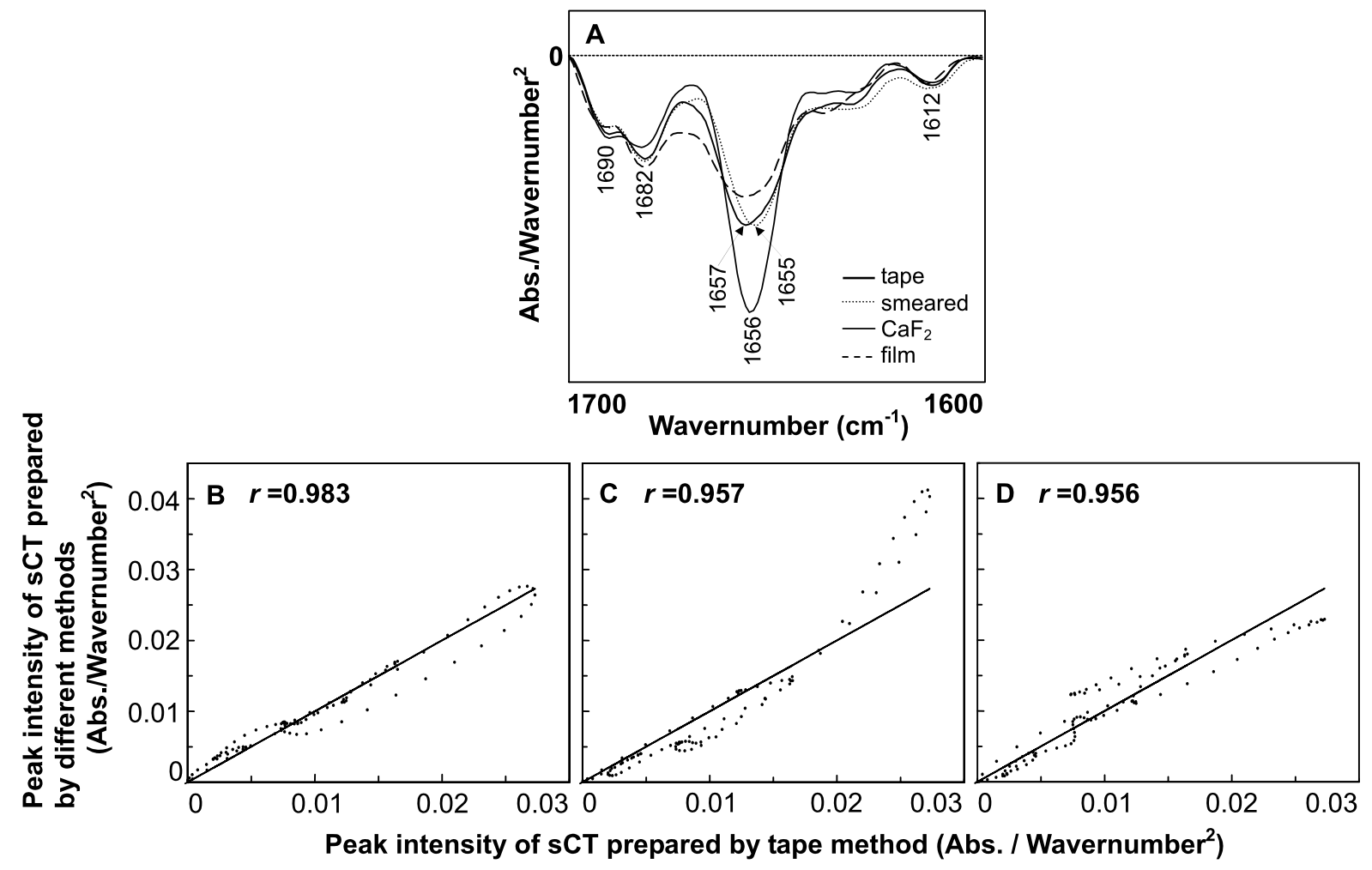

Fig. 2. Second-derivative amide I spectra of sCT prepared by different methods (A) and correlation of peak intensity of sCT prepared by tape method with that of the peak intensity of $\mathrm{SCT}$ prepared by smeared, $\mathrm{CaF}_{2}$ or film method using the second-derivative spectra in amide I region (B-D). Key: (A) baseline corrected and area-normalized spectra; (B) tape method vs. smeared method; (C) tape method vs. $\mathrm{CaF}_{2}$ method; (D) tape method vs. film method. 
This was accomplished by calculating the correlation coefficient $(r)$ for the spectrum of sCT by using tape preparation method as a reference. It is apparent that the calculated $r$ value was 0.983 for smeared method vs. tape method, 0.957 for $\mathrm{CaF}_{2}$ method vs. tape method, or 0.956 for film method vs. tape method, respectively (Fig. 2B-D). The correlation coefficients were very close to unity, suggesting no appreciable structural change. In other word, four sample preparation methods used here did not cause the additional sCT structural alterations, suggesting that these four sample preparation methods may be suitable for studying the protein secondary structure whether in powder or film form. Because the sCT powder was a native form (tape method) and the $r$ value of smeared method was higher than that of the $\mathrm{CaF}_{2}$ method or film method, indicating that by smearing sCT powder on the surface $\mathrm{KBr}$ pellet (smeared method) was the best optimal sample preparation method.

\section{Acknowledgements}

This work was supported by National Science Council, Taipei, Taiwan, Republic of China (97-2628B-264-001-MY3).

\section{References}

[1] A. Barth, Infrared spectroscopy of proteins, Biochim. Biophys. Acta 1767 (2007), 1073-1101.

[2] C.H. Chesnut 3rd, M. Azria, S. Silverman, M. Engelhardt, M. Olson and L. Mindeholm, Salmon calcitonin: a review of current and future therapeutic indications, Osteoporos. Int. 19 (2008), 479-491.

[3] A. Dong, P. Huang and W.S. Caughey, Protein secondary structures in water from second-derivative amide I infrared spectra, Biochemistry 29 (1990), 3303-3308.

[4] S. Frokjaer and D.E. Otzen, Protein drug stability: a formulation challenge, Nat. Rev. Drug Discov. 4 (2005), 298-306.

[5] P.I. Haris and D. Chapman, Analysis of polypeptide and protein structures using Fourier transform infrared spectroscopy, Methods Mol. Biol. 22 (1994), 183-202.

[6] K.A. Henzler-Wildman, D.K. Lee and A. Ramamoorthy, Determination of alpha-helix and beta-sheet stability in the solid state: a solid-state NMR investigation of poly (L-alanine), Biopolymers 64 (2002), 246-254.

[7] L. Jorgensen, E.H. Moeller, M. van de Weert, H.M. Nielsen and S. Frokjaer, Preparing and evaluating delivery systems for proteins, Eur. J. Pharm. Sci. 29 (2006), 174-182.

[8] M.C. Lai and E.M. Topp, Solid-state chemical stability of proteins and peptides, J. Pharm. Sci. 88 (1999), 489-500.

[9] Y.H. Lee and P.J. Sinko, Oral delivery of salmon calcitonin, Adv. Drug Deliv. Rev. 42 (2000), 225-238.

[10] J.G. Lees and R.W. Janes, Combining sequence-based prediction methods and circular dichroism and infrared spectroscopic data to improve protein secondary structure determinations, BMC Bioinformatics 9 (2008), 24.

[11] S.Y. Lin and J.L. Chien, In vitro simulation of solid-solid dehydration, rehydration, and solidification of trehalose dihydrate using thermal and vibrational spectroscopic techniques, Pharm. Res. 20 (2003), 1926-1931.

[12] S.Y. Lin and H.L. Chu, Fourier transform infrared spectroscopy used to evidence the prevention of beta-sheet formation of amyloid beta(1-40) peptide by a short amyloid fragment, Int. J. Biol. Macromol. 32 (2003), 173-177.

[13] S.Y. Lin, C.J. Ho and M.J. Li, Thermal stability and reversibility of secondary conformation of alpha-crystallin membrane during repeated heating processes, Biophys. Chem. 74 (1998), 1-10.

[14] S.Y. Lin, Y.S. Wei, T.F. Hsieh and M.J. Li, Pressure dependence of human fibrinogen correlated to the conformational alpha-helix to beta-sheet transition: an Fourier transform infrared microspectroscopic study, Biopolymers 75 (2004), 393402.

[15] Y.F. Maa and S.J. Prestrelski, Biopharmaceutical powders: particle formation and formulation considerations, Curr. Pharm. Biotechnol. 1 (2000), 283-302.

[16] M.C. Manning, Use of infrared spectroscopy to monitor protein structure and stability, Expert Rev. Proteomics 2 (2005), 731-743.

[17] N.M. Mehta, A. Malootian and J.P. Gilligan, Calcitonin for osteoporosis and bone pain, Curr. Pharm. Des. 9 (2003), 2659-2676.

[18] J.T. Pelton and I.R. McLean, Spectroscopic methods for analysis of protein secondary structure, Anal. Biochem. 277 (2000), 167-176. 
[19] S.J. Prestrelski, N. Tedeschi, T. Arakawa and J.F. Carpenter, Dehydration-induced conformational transitions in proteins and their inhibition by stabilizers, Biophys. J. 65 (1993), 661-671.

[20] B. Rost, Review: protein secondary structure prediction continues to rise, J. Struct. Biol. 134 (2001), 204-218.

[21] H. Susi and D.M. Byler, Protein structure by Fourier transform infrared spectroscopy: second derivative spectra, Biochem. Biophys. Res. Commun. 115 (1983), 391-397.

[22] R. Tantipolphan, T. Rades and N.J. Medlicott, Insights into the structure of protein by vibrational spectroscopy, Curr. Pharm. Anal. 4 (2008), 53-68.

[23] E. Vass, M. Hollósi, F. Besson and R. Buchet, Vibrational spectroscopic detection of beta- and gamma-turns in synthetic and natural peptides and proteins, Chem. Rev. 103 (2003), 1917-1954.

[24] W. Wang, Lyophilization and development of solid protein pharmaceuticals, Int. J. Pharm. 203 (2000), 1-60.

[25] R.Y. Yada, R.L. Jackman and S. Nakai, Secondary structure prediction and determination of proteins - a review, Int. J. Pept. Protein Res. 31 (1988), 98-108. 


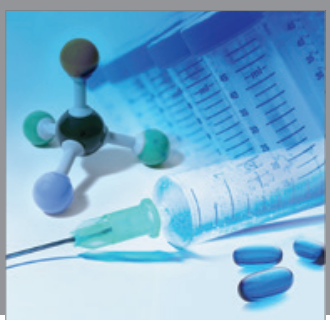

International Journal of

Medicinal Chemistry

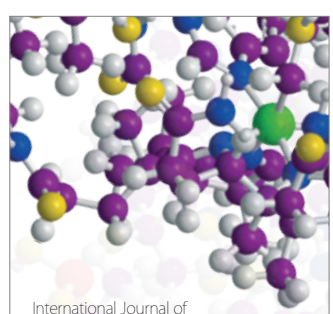

Carbohydrate Chemistry

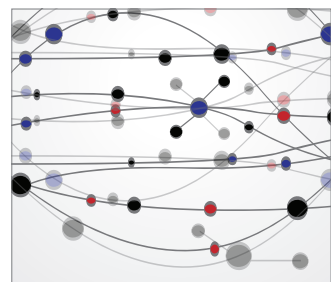

The Scientific World Journal
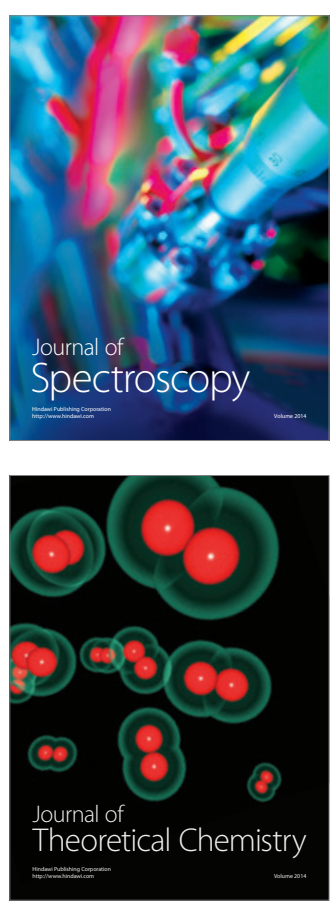
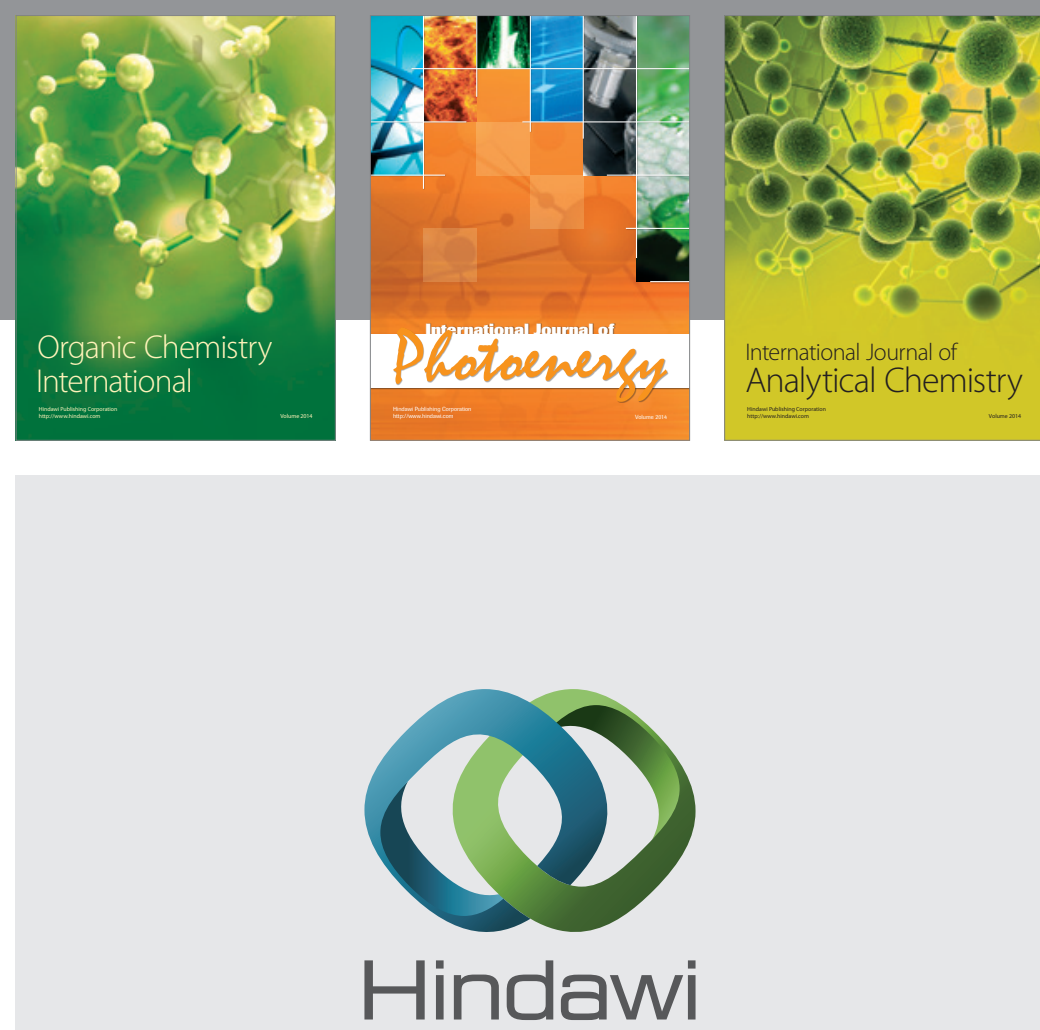

Submit your manuscripts at

http://www.hindawi.com
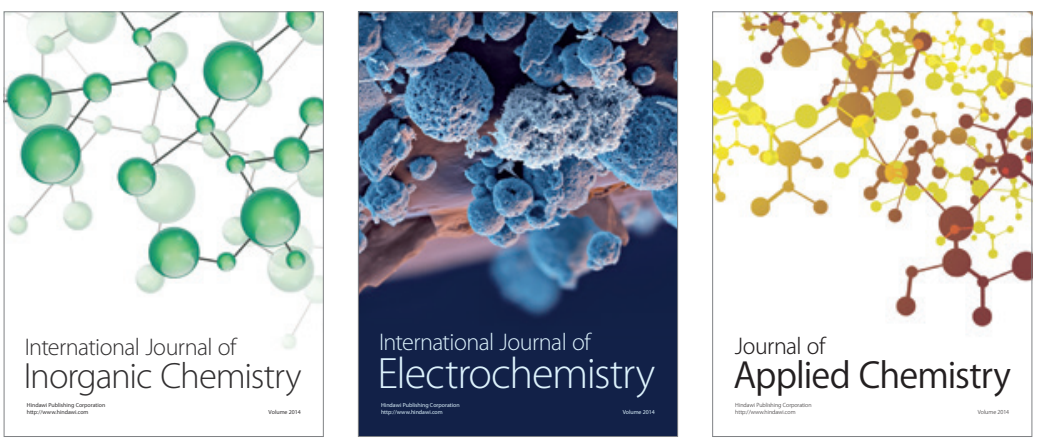

Journal of

Applied Chemistry
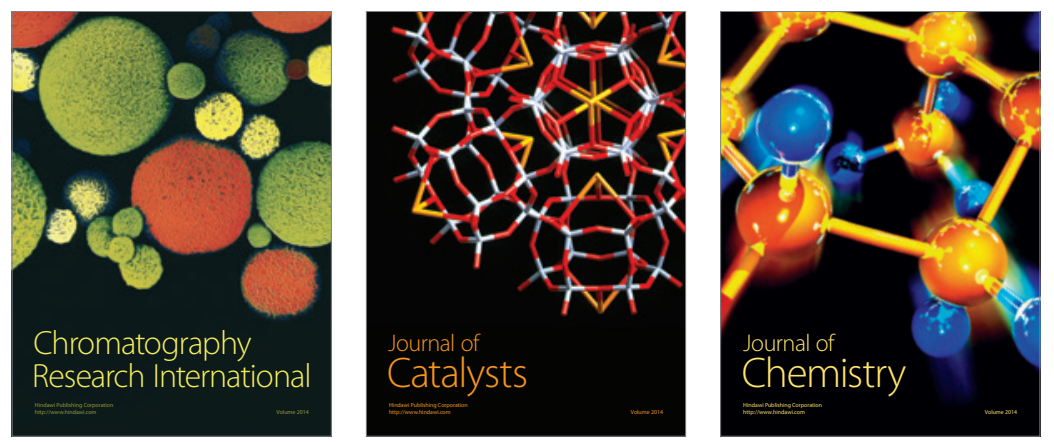
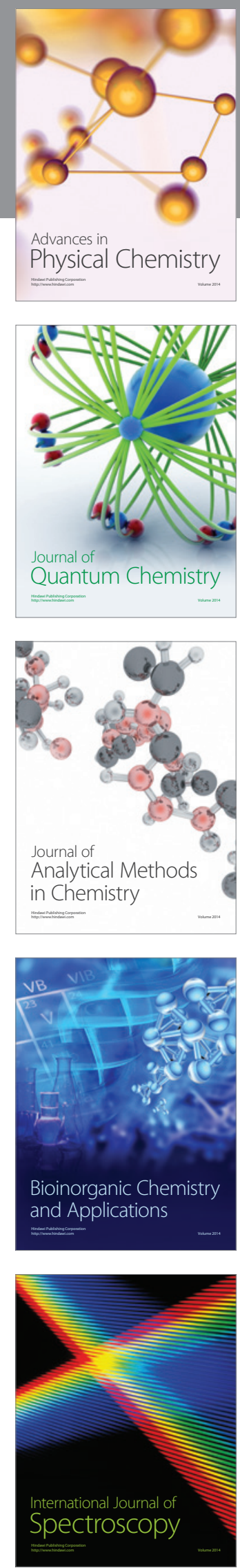